\title{
WORKING FIELD THEORY PROBLEMS WITH RANDOM WALKS
}

By:

K.R. Davey

COMPEL, ISSN 0332-1649, vol. 24, no. 1, 2005, pp. 229-240

PR - 387

Center for Electromechanics

The University of Texas at Austin

PRC, Mail Code R7000

Austin, TX 78712

(512) $471-4496$ 


\section{Working field theory problems with random walks}

\section{Working field theory problems}

\author{
Kent R. Davey
}

Center for Electromechanics, University of Texas, Austin, Texas, USA

\begin{abstract}
Purpose - The purpose of this paper is to demonstrate how Monte Carlo methods can be applied to the solution of field theory problems.

Design - This objective is achieved by building insight from Laplacian field problems. The point solution of a Laplacian field problem can be viewed as the solid angle average of the Dirichlet potentials from that point. Alternatively it can be viewed as the average of the termination potential of a number of random walks. Poisson and Helmholtz equations add the complexity of collecting a number of packets along this walk, and noting the termination of a random walk at a Dirichlet boundary.

Findings - When approached as a Monte Carlo problem, Poisson type problems can be interpreted as collecting and summing source packets representative of current or charge. Helmholtz problems involve the multiplication of packets of information modified by a multiplier reflecting the conductivity of the medium.

Practical implications - This method naturally lends itself to parallel processing computers.

Originality/value - This is the first paper to explore random walk solutions for all classes of eddy current problems, including those involving velocity. In problems involving velocity, the random walk direction enters depending on the walk direction with respect to the local velocity.
\end{abstract}

Keywords Finite difference methods, Monte Carlo methods, Random functions

Paper type Technical paper

\section{Introduction}

Finite element and boundary element methods now dominate the industry as the methods of choice for solving field theory problems. Monte Carlo methods were introduced over 58 years ago (Katatuni, 1944), but still await the strong advent of parallel processing computers before they will be competitive with the more classical direct methods. This paper discusses a different way of approaching field theory problems, the bulk of which depend on random numbers. The work has three motivations.

(1) To discuss an analysis approach, which lends itself to what will undoubtedly be the trend in computers - parallel processing. There are some fundamental barriers to increasing the processor speed, but seemingly less so than the number of parallel processors.

(2) To offer a method that gives the field theoretician yet another way of thinking about a field problem, hopefully one that will add additional intuitive insight into their expected solutions.

(3) To generalize the theory to a broad class of field theory problems. In researching the subject in the IEEE database, it seems that only one paper

COMPLL: The International Journal for Computation and Mathematics in Electrical and Electronic Engineering Vol. 24 No. 1,2005
pp. 229.237

Emerald Group Publishing Limited DOl 10.1108/03321640510571255 
COMPEL 24,1 discussed application of the technique to eddy current problems (Davey and Nair, 1993), and none have attempted to generalize the theory to eddy problems with motion.

The number of papers discussing the use of the Monte Carlo method in the actual calculation of electric and magnetic fields are few. Micu (1996) discusses a simple application of the method to electrostatic problems, and Mandayam et al. (1996) discuss its application to a magnetostatic problem. Sadiku (1990) discusses it under the variant of the fixed aleatory route.

The three classes of problems to be discussed are Laplacian, Poisson, and Helmholtz. These universal formulations have not been worked out before, especially for generalized eddy problems involving velocity. A universal formulation to any $3 \mathrm{D}$ problem will be presented for the Laplacian problem. Then the formulation of Poisson and Helmholtz problems will be presented. These formulations remain the same regardless of the dimensionality of the problem. Given that working hypothesis, specific examples will focus on one dimensional problems for the second two classes of problems.

\section{Laplacian problems}

$$
\nabla^{2} \boldsymbol{\Phi}=0
$$

The problem has been considered by Micu (1996) and Mandayam et al. (1996) through electrostatic and magnetostatic examples. Consider first that the solution of any three dimensional Laplacian problem can be thought of as the weighted sum of the potentials as seen through the solid angle subtended from the border surfaces to the desired field point. Consider the two dimensional problem shown in Figure 1. The potential $\boldsymbol{\Phi}_{0}$ is related to the perimeter potentials as

$$
\boldsymbol{\Phi}_{0}=\frac{1}{2 \pi} \sum_{k=1}^{6} \beta_{k} \boldsymbol{\Phi}_{k} .
$$

If one of the borders has a Neumann boundary condition as shown in Figure 2, the border acts identically to a mirror as in the lower inset. The potential at the center of the original figure will be

$$
\boldsymbol{\Phi}_{0}=\frac{1}{\left(2 \pi-\beta_{6}\right)} \sum_{k=1}^{5} \beta_{k} \boldsymbol{\Phi}_{k} .
$$

At any other position it will reflect the weighted sum of the potential over the mirror segment.

\subsection{Random walk}

Equations (2) and (3) can be replicated numerically through the concept of random walks. Consider starting at the field point, and randomly "stepping" in any direction until a boundary is reached. The potential at the starting point is simply the average of the potentials of the end points of many random walks. Accuracy is improved by both increasing the number of random walks and decreasing the step size.

Implementation of a random walk necessarily implies the existence of a grid of "steps". The steps can be randomly placed. If the placement of the steps is random, 


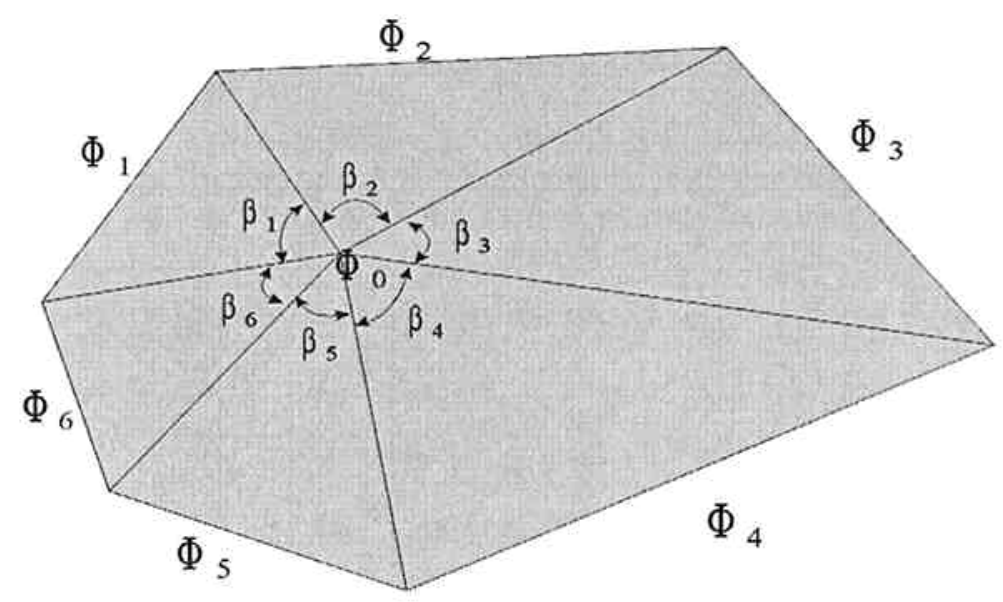

\section{Working field theory problems}

Figure 1. The field point potential is related to the perimeter potentials through the angle subtended

then a table must be constructed for every point and its nearest neighbors. Nine nearest neighbors are required in 3D problems, while five are required in 2D. Using the guidelines of Girdinio et al. (1983), equation (1) can be written at point zero as

$$
\boldsymbol{\Phi}_{0}=\sum_{k=1}^{9} p_{k} \boldsymbol{\Phi}_{k} .
$$

Using Taylor's expansion, the potential at point 1 can be expressed in terms of the potential at point 0 as

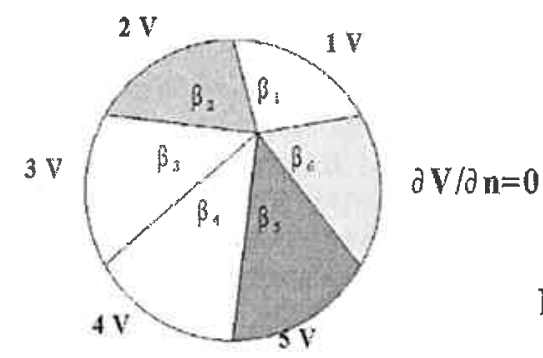

\section{Neumann Problem}

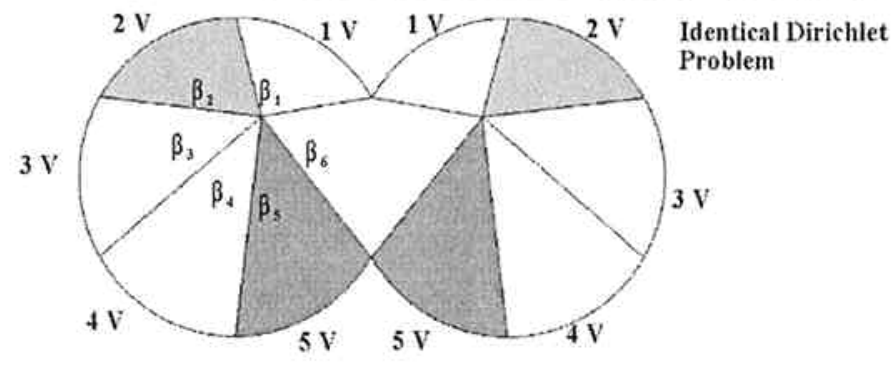

Figure 2. A Neumann boundary condition will always act like a mirror 
COMPEL 24,1

$$
\boldsymbol{\Phi}_{1}=\boldsymbol{\Phi}_{0}+\frac{\partial \boldsymbol{\Phi}_{0}}{\partial x} \delta x_{1}+\cdots+\frac{\partial^{2} \boldsymbol{\Phi}_{0}}{\partial z^{2}} \delta z_{1}^{2}+\cdots+\frac{\partial^{2} \boldsymbol{\Phi}_{0}}{\partial y \partial z} \delta y_{1} \delta z_{1},
$$

where $\partial x_{1}=x_{1}-x_{0}$. Proceeding in this way it is possible to write a matrix equation as in equation (6). Designating the matrix multiplier as A, and noting that rows 2, 3 , and 4 correspond to the Laplacian operator components, $\partial^{2} \boldsymbol{\Phi} / \partial x^{2}, \partial^{2} \boldsymbol{\Phi} / \partial y^{2}$, and $\partial^{2} \boldsymbol{\Phi} / \partial z^{2}$, $p$ in equation (4) would follow as in equation (7).

$$
\left[\begin{array}{ccccccc}
\delta x_{1} & \delta x_{1}^{2} & \delta y_{1}^{2} & \delta z_{1}^{2} & \delta y_{1} & \ldots & \delta y_{1} \delta z_{1} \\
\delta x_{2} & \delta x_{2}^{2} & \delta y_{2}^{2} & \delta z_{2}^{2} & \delta y_{2} & \ldots & \delta y_{2} \delta z_{2} \\
\vdots & \vdots & \vdots & \vdots & \vdots & \vdots & \vdots \\
\delta x_{9} & \delta x_{9}^{2} & \delta y_{9}^{2} & \delta z_{9}^{2} & \delta y_{9} & \ldots & \delta y_{9} \delta z_{9}
\end{array}\right]\left[\begin{array}{c}
\frac{\partial \Phi}{\partial x} \\
\frac{\partial^{2} \Phi}{\partial x^{2}} \\
\vdots \\
\frac{\partial^{2} \Phi}{\partial y \partial z}
\end{array}\right]=\left[\begin{array}{c}
\Phi_{1}-\boldsymbol{\Phi}_{0} \\
\boldsymbol{\Phi}_{2}-\boldsymbol{\Phi}_{0} \\
\vdots \\
\boldsymbol{\Phi}_{9}-\boldsymbol{\Phi}_{0}
\end{array}\right] .
$$

For an equally equal spaced finite difference grid, all the $p$ s are equal to $1 / 6$, but in general they will all be different. The general probability that the walk will progress to position $k$ is

$$
p_{k}=\frac{A^{-1}(2, k)+A^{-1}(3, k)+A^{-1}(4, k)}{\sum_{j=1}^{9}\left\{A^{-1}(2, j)+A^{-1}(3, j)+A^{-1}(4, j)\right\}} .
$$

The walk progresses by consecutively collecting random numbers and moving to one of nine local positions. Designate the sum of the $p \mathrm{~s}$ as $P_{\mathrm{SUM}}$. If the random number is between 0 and $p_{1} / P_{\text {SUM }}$, the walk progresses to local position 1 ; if it is between $p 1$ and $\left(p_{1}+p_{2}\right) / P_{\text {SUM }}$, the walk progresses to local position 2 . The potential is registered when a Dirichlet boundary is reached. The average of these ending points is the solution sought. Material changes result merely in a local change in probability. In a magnetostatic problem, the probability of moving into a permeable medium is decreased by the multiplier $2 \mu /\left(\mu_{0}+\mu\right)$, and conversely increased in the direction outward from the medium by $2 \mu_{0} /\left(\mu_{0}+\mu\right)$. This is a straightforward process, but one that gets a lot more interesting with Helmholtz and Poisson problems. These principles apply to electrostatic problems using the dual relation $\varepsilon \propto 1 / \mu$.

Before proceeding to the more interesting problems, note that modern tools speed up what appears to be a tedious process. One never executes the problem as described in practice. Linear arrays of random numbers, combined with cumulative sums, are employed to speed this up. Why these cumulative sums are necessary will become clearer in the next section.

\section{Poisson problems}

Consider the Poisson problem governing electric potential in a charge filled region,

$$
\nabla^{2} \Phi=-\frac{\rho}{\varepsilon}
$$


The nonzero right hand side necessitates the collection of what will be called packets along the walk. In a one dimensional problem, equation (9) would be discretized with an even grid as

$$
\boldsymbol{\Phi}_{0}=\left(\frac{\boldsymbol{\Phi}_{1}+\boldsymbol{\Phi}_{-1}}{2}\right)+\delta x^{2} \frac{\rho}{2 \varepsilon} .
$$

As before, every walk begins at an arbitrary point and ends at a Dirichlet wall. An $n$ step walk value is the sum of the number of packets plus the potential $\Phi_{n}$ at the end of the walk,

$$
R_{\text {walk } m}=\sum_{\text {step } i=1}^{n} \frac{\rho \delta x^{2}}{2 \varepsilon}+\Phi_{n}
$$

The mean value $\left\langle R_{\text {walks }}\right\rangle$ is the potential at the starting location. Suppose there are $k$ discrete positions, with $k$ being odd. If $\rho$ is uniform then the number of packets collected starting at the center position is $((k-1) / 2)^{2}$. The positions adjacent to the center would have $((k-1) / 2)^{2}-1$ packets, $((k-1) / 2)^{2}-3$, etc. If the number of positions is 11 , and one starts the random walk at the middle at $k=6$, the average number of packets collected versus number of walks is shown in Figure 3. The exact number should be $((11-1) / 2)^{2}=25$. If $k$ is even, then the packets collected from the center, out is $(k / 2)^{2}-k / 2,(k / 2)^{2}-k / 2-2,(k / 2)^{2}-k / 2-4$, etc. Matlab allows the definition of matrices of random numbers. These can easily be translated to random strings of ones and minus ones. Using cumulative sums, one can determine when a boundary is reached and how many steps were required.

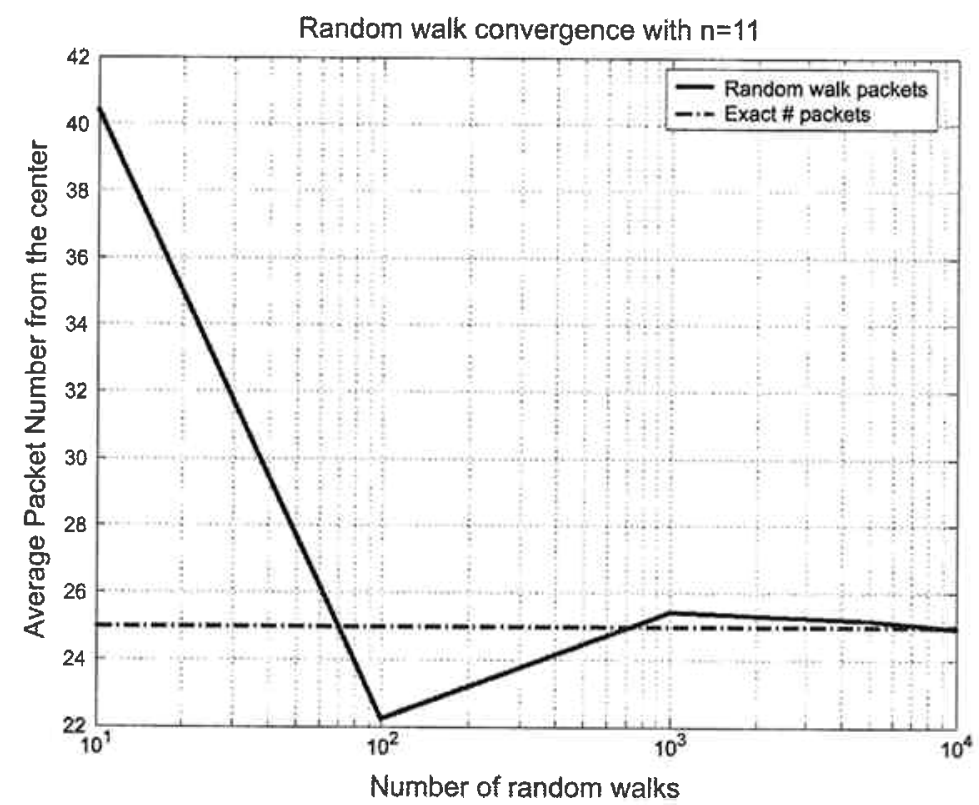

Figure 3. Average number of packets collected versus random walk size 
COMPEL 24,1

234
4. Helmholtz equations

Consider the Helmholtz equation,

$$
\nabla^{2} \boldsymbol{\Phi}-k^{2} \boldsymbol{\Phi}=-\frac{\rho}{\varepsilon}
$$

whereas Poisson equations summed the packets collected, the Helmholtz equation takes the product of the packets collected.

The discretized form of this equation is

$$
\Phi_{0}=\frac{\Phi_{1}+\Phi_{-1}+\delta x^{2} \frac{\rho}{\varepsilon}}{2\left(1+\frac{k^{2} \delta x^{2}}{2}\right)}
$$

Consider first the condition when $\rho=0$. An $n$ step walk is now the product of the number of packets times the potential at the end of the walk,

$$
R_{\text {walk } m}=\boldsymbol{\Phi}_{n} \prod_{\text {step } i=1}^{n} \frac{1}{\left(1+\frac{k^{2} \delta x^{2}}{2}\right)} .
$$

As before, the potential is the mean of this value $R$ for all random walks.

Consider a one dimensional problem with $a<x<b, a=0, b=0.75, \Phi a=1$, $\Phi b=7$, and $k=3$. Shown in Figure 4 is the random walk prediction against the exact solution for a number of walk sizes. Even a modest walk size of 50 yields reasonable results.

When $\rho \neq 0$, the solution procedure is complicated by the fact that both a sum and a product of the packets must be collected. In particular the product quantity involving

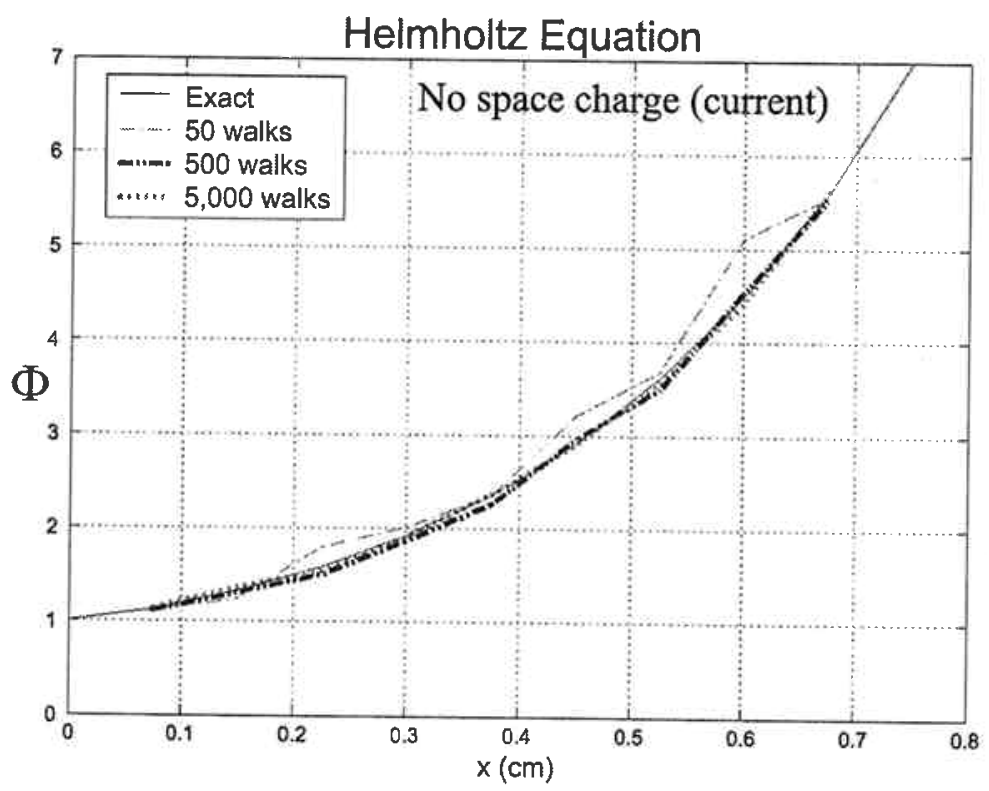

Figure 4.

Helmholtz equation solution with variable number of walks 
$1 /\left(1+k^{2} \delta x^{2} / 2\right)$ must be multiplied by $\rho / \varepsilon$ at every step. Consider again an $n$ step walk, i.e., the walk ending at a Dirichlet wall at step $n$. Let $m$ be the position at some point prior to $n$. The $n$ step walk is now computed as

$$
R_{\mathrm{walk} m}=\boldsymbol{\Phi}_{n} \prod_{\text {step } i=1}^{n} \frac{1}{\left(1+\frac{k^{2} \delta x^{2}}{2}\right)}+\left\{\sum_{i=1}^{n} \frac{\delta x^{2} \rho}{2 \varepsilon} \prod_{\text {step } j=1}^{i} \frac{1}{\left(1+\frac{k^{2} \delta x^{2}}{2}\right)}\right\} .
$$

Consider now parameters similar to the previous case, but with $k=4$, and $\rho / \varepsilon=70$. The exact solution now becomes "S" shaped. Figure 5 shows the result of this computation with 30,300 , and 1,000 random walks. A modest number of walks again predicts the solution reasonably well. Assuming equal step sizes, the " 2 " becomes " 4 " and " 6 " for two and three dimensional problems, respectively.

\section{Velocity effects}

Consider a magnetic field problem in two dimensions with a conducting body moving with a velocity $v$. The governing equation for the vector potential in a time harmonic problem can be written as

$$
\nabla^{2} \vec{A}-k^{2} \vec{A}+\mu \sigma \vec{v} \times \nabla \times \vec{A}=-\mu \vec{J}
$$

where $k^{2}=j \omega \mu \sigma$. In a one dimensional embodiment, this equation can be written as

$$
\frac{\mathrm{d}^{2} A}{\mathrm{~d} x^{2}}-k^{2} A-\mu \sigma \nu \frac{\mathrm{d} A}{\mathrm{~d} x}=-\mu J .
$$

The discretized form of this equation yields the result

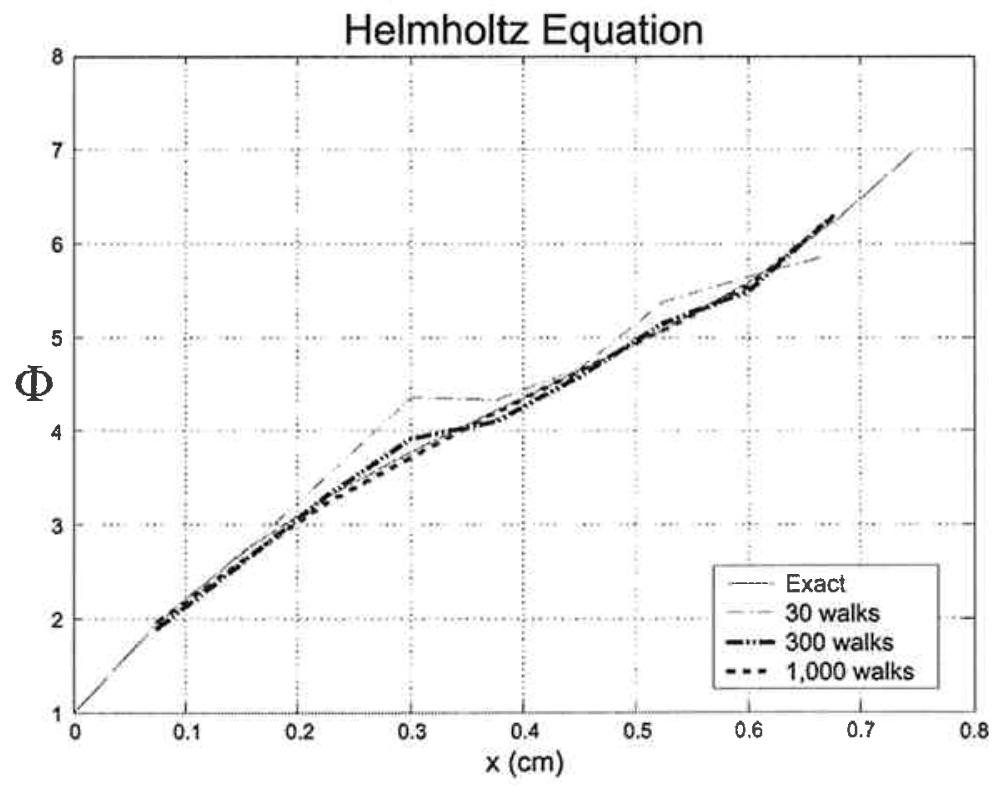

Figure 5.

Prediction of a Helmholtz problem with non-zero charge density 
COMPEL

24,1

236

$$
A_{0}=\frac{\left\{A_{1}\left(1-\frac{\mu \sigma v}{2} \delta x\right)+A_{-1}\left(1+\frac{\mu \sigma v}{2} \delta x\right)+\mu J \delta x^{2}\right\}}{2\left(1+\frac{k^{2} \delta x^{2}}{2}\right)} .
$$

The random walk requires summing the packets involving $\mu J$, again with the multiplier $\left(1+k^{2} \delta x^{2} / 2\right)$. The random walk is

$$
R_{\text {walk } m}=A_{n} \prod_{\text {step } i=1}^{n} \frac{\left(1 \pm \frac{\mu \sigma v}{2} \delta x\right)}{\left(1+\frac{k^{2} \delta x^{2}}{2}\right)}+\sum_{i=1}^{n}\left\{\frac{\mu J \delta x^{2}}{2} \prod_{\text {step } j=1}^{i} \frac{\left(1 \pm \frac{\mu \sigma v}{2} \delta x\right)}{\left(1+\frac{k^{2} \delta x^{2}}{2}\right)}\right\} .
$$

The multiplier has a \pm sign. If the random step is to the right, a minus sign is employed, while if to the left, a plus sign.

Let $\mu J=100, \mu \circ v=24$, and $k^{2}=3$. The results of three random walks are shown in Figure 6. The direction dependent sign, and the results argue for a larger number of walks for the same accuracy.

\section{Conclusions}

Monte Carlo methods are useful in providing a new way of looking at field theory problems. The collection of packets with appropriate multipliers has proven to be one approach applicable to a range of problems. When the random walk is sensitive to direction, as is the case with velocity problems, the number of random walks required for an accurate solution will increase considerably. These techniques are suited to parallel processing, and thus may find greater use as parallel processing advances.

Figure 6.

Random walk predictions with velocity

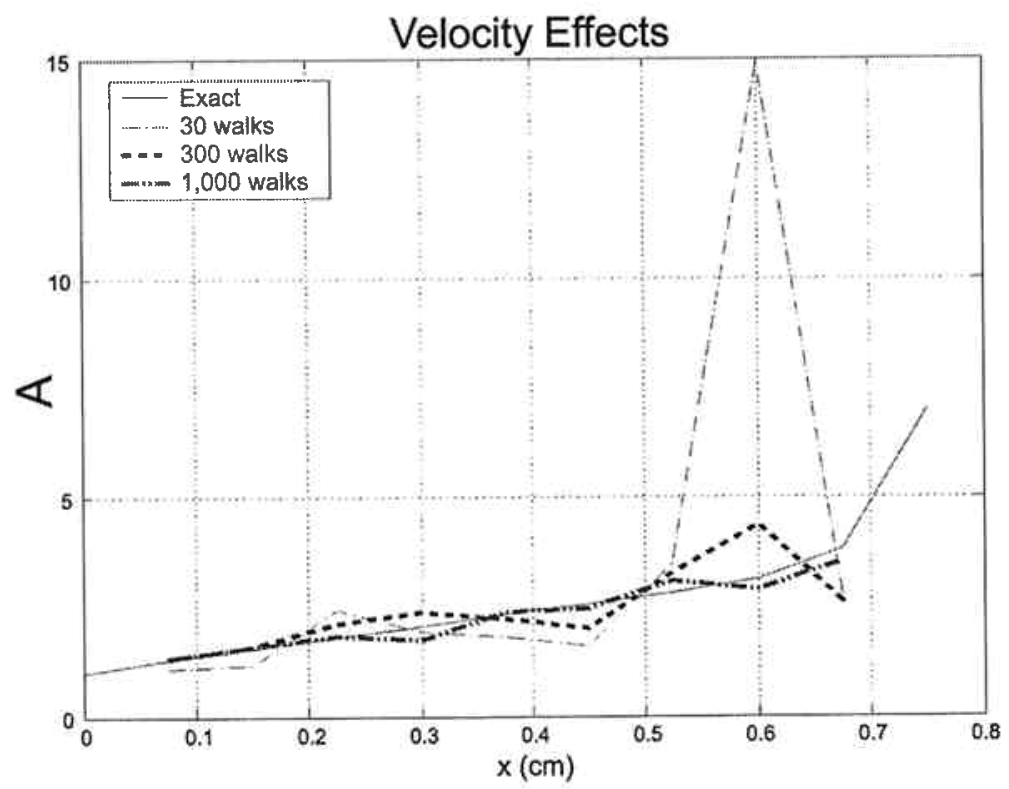




\section{References}

Micu, D. (1996), "Numerical synthesis of electrostatic fields by the Monte Carlo method", IEEE Transactions of Magnetics, Vol. 29 No. 2, pp. 1966-9.

Davey, K.R. and Nair, M.B. (1993), "A Monte Carlo technique for solving eddy current problems", IEEE Transactions on Magnetics, Vol. 29 No. 2, pp. 1376-9.

Girdinio, P., Molfino, P., Molinari, G., Puglisi, L. and Viviani, A. (1983), "Finite difference discretization procedures with improved continuity of interpolation functions", IEEE Trans. Magn., Vol. MAG-19 No. 6, pp. 2558-61.

Katatuni, S. (1944), "Two dimensional Brownian motion and harmonic functions", Proceedings of the Imperial Academy of Japan, Vol. XX No. 9 pp. 706-14.

Mandayam, S., Upta, L., Upda, S.S. and Lord, W. (1996), "Monte Carlo methods for modeling magnetostatic NDE phenomena: a feasibility study", IEEE Transactions on Magnetics, Vol. 32 No. 3, pp. 1425-7.

Sadiku, M.N.O. (1990), "Monte Carlo methods in an introductory electromagnetic course", IEEE Trans. Educ., Vol. 33 No. 1, pp. 73-9. 